\title{
Merit2U: an IoT-based merit point management system for university students' hostel application
}

\begin{abstract}
Merit2U is a web-based system to manage merit points for students' college application. This system provides better merit point management where the points are calculated automatically and electronically using web-based application.
\end{abstract}

Keyword: Merit system; Internet of Things; Web application; QR code 